Portland State University

PDXScholar

Electrical and Computer Engineering Faculty

Publications and Presentations

$1-1-2008$

\title{
The Copula: A Tool for Simulating Speckle Dynamics
}

Donald D. Duncan

Portland State University

Sean J. Kirkpatrick

Follow this and additional works at: https://pdxscholar.library.pdx.edu/ece_fac

Part of the Electrical and Computer Engineering Commons

Let us know how access to this document benefits you.

\section{Citation Details}

Donald D. Duncan and Sean J. Kirkpatrick, "The copula: a tool for simulating speckle dynamics," J. Opt. Soc. Am. A 25, 231-237 (2008)

This Article is brought to you for free and open access. It has been accepted for inclusion in Electrical and Computer Engineering Faculty Publications and Presentations by an authorized administrator of PDXScholar. Please contact us if we can make this document more accessible: pdxscholar@pdx.edu. 


\title{
The copula: a tool for simulating speckle dynamics
}

\author{
Donald D. Duncan* and Sean J. Kirkpatrick \\ Department of Biomedical Engineering, Oregon Health and Science University, 3303 SW Bond Avenue, Portland, \\ Oregon 97239, USA \\ *Corresponding author: donald.duncan@bme.ogi.edu
}

Received August 21, 2006; revised October 26, 2007; accepted November 6, 2007; posted November 13, 2007 (Doc. ID 86711); published December 20, 2007

Use of a copula for generating a sequence of correlated speckle patterns is introduced. The chief characteristic of this algorithm is that it generates a continuous speckle sequence with a specified evolution of the correlation and does so with just two arrays of random numbers. Thus, physically realistic temporally varying speckle patterns with proper first- and second-order statistics are easily realized. We illustrate use of the algorithm for generating sequences with prescribed Gaussian, exponential, and equal-interval correlations and demonstrate how correlation times can be specified independently. This approach to generating sequences of random realizations with prescribed correlations should prove useful in modeling such phenomena as dynamic light scatter, flow-dependent laser speckle contrast, and propagation of spatial coherence. (c) 2007 Optical Society of America

OCIS codes: $030.6140,030.6600,110.4500,110.6150,120.6150,300.6480$.

\section{INTRODUCTION}

The speckle phenomenon is observed in any coherent imaging modality such as synthetic aperture radar, optical coherence tomography, ultrasound, or any number of nonimaging measurement schemes involving laser illumination. Quantitative interpretation of the data from such measurement schemes (whether imaging or nonimaging) often hinges on accurate knowledge of the statistical behavior of the speckle phenomenon. To complement experimental measurements, researchers often turn to computer simulation of the phenomenon of interest. One important situation is the temporal decorrelation of a speckle pattern. Such a behavior is of interest, for example, in the use of laser speckle dynamics to assess fluid flow or in quasi-elastic light scatter to determine molecular mass.

Various means of generating dynamic speckle pattern sequences have been proposed. For example, Rabal et al. [1] used a fast Fourier transform (FFT) technique involving manipulation of the phase of the scattering particles. Specifically, they explored the effects of coordinated and uncoordinated out-of-plane motion. Other authors have used a similar approach using the FFT but taking a statistical approach to the generation of speckle sequences [2,3]. These authors based the evolution of the speckle patterns on manipulating the variance of the phase. The basis for their approach was the complex Gaussian moment theorem [4], and thus only speckle pattern sequences with monotonically decreasing correlations could be synthesized.

Here, we take a different approach that provides for the generation of a sequence of speckle patterns with an arbitrary (not necessarily monotonic) correlation function. This approach, based on a statistically rigorous evolution between two statistically independent speckle patterns, uses the concept of a copula [5]. We discuss the theory of such a concept and illustrate its use in producing speckle decorrelation sequences that correspond to unordered (e.g., Brownian) as well as ordered motion [6].

\section{THEORY}

The word "copula" is from the Latin meaning "to bond." In the context of probability theory it has come to be defined as a function that links individual marginal distributions into a joint, multivariate distribution. The basis for this idea is Sklar's theorem [5], which states that given a joint (cumulative) distribution function, $H(x, y)$, and the marginal (cumulative) distribution functions, $F(x)$ and $G(y)$, there exists a (copula), $C$, such that

$$
H(x, y)=C[F(x), G(y)] .
$$

The copula plays the role of linking two marginal distribution functions into a prescribed joint distribution function. Sklar's theorem [5] further states that if the distribution functions are continuous, then the copula is unique.

In the material that follows, we use a Gaussian copula (there are many other types [5]) to correlate two initially statistically independent (S.I.) uniformly distributed random variables (RVs), and subsequently use the resulting correlated samples to create sequences of correlated speckle patterns.

\section{A. Phase Generation}

We begin with two uniformly distributed, statistically independent RVs, $X_{1}$ and $X_{2}$. From this pair, the BoxMueller transformation [7] produces a new pair of RVs,

$$
Y_{1}=\mu+\sigma \sqrt{-2 \ln X_{1}} \cos \left(2 \pi X_{2}\right),
$$




$$
Y_{2}=\mu+\sigma \sqrt{-2 \ln X_{1}} \sin \left(2 \pi X_{2}\right),
$$

such that the means and variances are given by

$$
E\left\{Y_{i}\right\}=\mu, \quad E\left\{\left(Y_{i}-E\left\{Y_{i}\right\}\right)^{2}\right\}=\sigma^{2},
$$

and $E$ denotes expectation. From this it follows that $Y_{1}$ and $Y_{2}$ are jointly normal, i.e., their joint probability density function is

$$
f_{Y_{1}, Y_{2}}=N\left(\mu_{1}, \mu_{2} ; \sigma_{1}, \sigma_{2} ; r\right)=N(\mu, \mu ; \sigma, \sigma ; 0),
$$

and because the correlation, $r$, is zero, they are S.I. (of course this statistical independence because of zero correlation is unique to the Gaussian distribution [7]). If we choose the specific case of $\mu=0, \sigma=1$ and use the following operations,

$$
\left|\begin{array}{c}
Z_{1} \\
Z_{2}
\end{array}\right|=\frac{1}{\sqrt{2}}\left|\begin{array}{cc}
1 & -1 \\
1 & 1
\end{array}\right|\left|\begin{array}{cc}
\sqrt{1+r} & 0 \\
0 & \sqrt{1-r}
\end{array}\right|\left|\begin{array}{l}
Y_{1} \\
Y_{2}
\end{array}\right|,
$$

we find that the RVs $Z_{1}$ and $Z_{2}$ are bivariate normal with correlation coefficient $r: f_{Z_{1}, Z_{2}}=N(0,0 ; 1,1 ; r)$. The BoxMueller transformation and the scaling and rotation constitute the copula that links the marginal distributions on $X_{1}$ and $X_{2}$ into the bivariate distribution on $Z_{1}$ and $Z_{2}$. Finally, we use the percentile transformation [7]

$$
T_{1}=F_{Z}\left(Z_{1}\right), \quad T_{2}=F_{Z}\left(Z_{2}\right),
$$

where $F_{Z}$ is the normal cumulative distribution function. From these operations we obtain the RVs $T_{1}$ and $T_{2}$, which are uniformly distributed on the unit interval and are no longer S.I. but have correlation coefficient $r$.

\section{B. Speckle Generation}

Having described a method by which one can arrive at a pair of uniformly distributed random variables with arbitrary correlation coefficient, we now proceed with a description of the generation of correlated objective (nonimaged) speckle patterns.

A spatially band-limited speckle pattern [8] can be synthesized easily by the following algorithm: Fill a circular region of diameter $D$ of a square matrix of dimension $L$ $\times L$ with complex numbers of unity amplitude and with phases uniformly distributed over $(0,2 \pi)$ (see Fig. 1). Upon Fourier transforming the $L \times L$ array and multiplying point-by-point by the complex conjugate, one arrives at a synthetic speckle pattern with exponential probability distribution. The position of the circular region within the larger array is irrelevant, as the Fourier shift theorem attests, and the ratio of $L$ to $D$ sets the minimum size

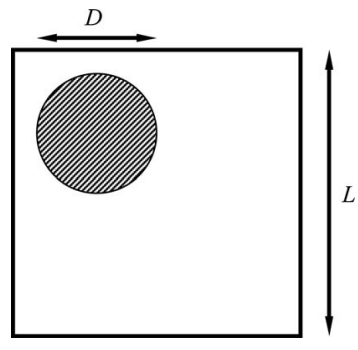

Fig. 1. Illustration of the synthetic speckle algorithm. Shaded region of the matrix is filled with complex numbers of unity amplitude and phases uniformly distributed over $(0,2 \pi)$. of the speckles. For example, if $L / D=2$, the Nyquist criterion is met and the smallest speckle is two pixels wide. This algorithm obviously results in a speckle pattern that fills the $L \times L$ array, but one typically uses only the $D$ $\times D$ portion of it. Application of the FFT in this algorithm accomplishes the many-to-one mapping observed in objective laser speckle patterns. Choice of a circular fill region within the larger array produces a statistically isotropic speckle pattern, but other patterns may be used as well.

Use of the phases $\phi_{1}=2 \pi t_{1} ; \phi_{2}=2 \pi t_{2}$ in this procedure, where $t_{1}$ and $t_{2}$ are uniformly distributed samples (instantiations of the corresponding $\mathrm{RVs}, T_{1}$ and $T_{2}$ ) as in the preceding discussion, produces a pair of correlated speckle patterns. The actual correlation between these two speckle patterns is given by

$$
\rho=\exp \left\{-\sigma_{\Delta \phi}^{2}\right\}
$$

where $\sigma_{\Delta \phi}^{2}=\operatorname{var}\left\{\phi_{1}-\phi_{2}\right\}$ is the variance of the phase difference. This result follows from the complex Gaussian moment theorem [4].

The evolution between the pair of speckle patterns as the correlation, $r$, is varied over the interval $(1,-1)$ is illustrated in Fig. 2. As shown in this sketch, for a specified correlation of $r=1$, the resulting phases are perfectly correlated and as a result, the two speckle patterns are identical. When $r=0$ the phases are uncorrelated and so too are the resulting speckle patterns. Interestingly, for negative correlations, $r \leq 0$, the phases display an anticorrelation, but because of the complex symmetric nature of the Fourier transform, the resulting speckle patterns are uncorrelated. Note that in the limit, $r=-1$, the phases are perfectly anticorrelated (a phase conjugate relationship between $2 \pi t_{1}$ and $2 \pi t_{2}$ ) and the resulting $L \times L$ speckle patterns (see discussion associated with Fig. 1) are $180^{\circ}$ rotations of one another.

As suggested by Fig. 2, we wish to create a sequence of speckle realizations over the interval $-1 \leq r \leq 1$, but our real interest is in the morphing of $Z_{1}$ from $Y_{1}$ to $-Y_{2}$ or of $Z_{2}$ from $Y_{1}$ to $Y_{2}$. For that we need to know the relationship between the specified correlation between $T_{1}$ and $T_{2}$ and the correlation between the realizations in the sequence, e.g., $T_{1}(1), \ldots, T_{1}(r), \ldots, T_{1}(-1)$. We denote the phase realizations in this sequence as $T_{1 k}, k=1,2, \ldots, N$, and find that this correlation is given by

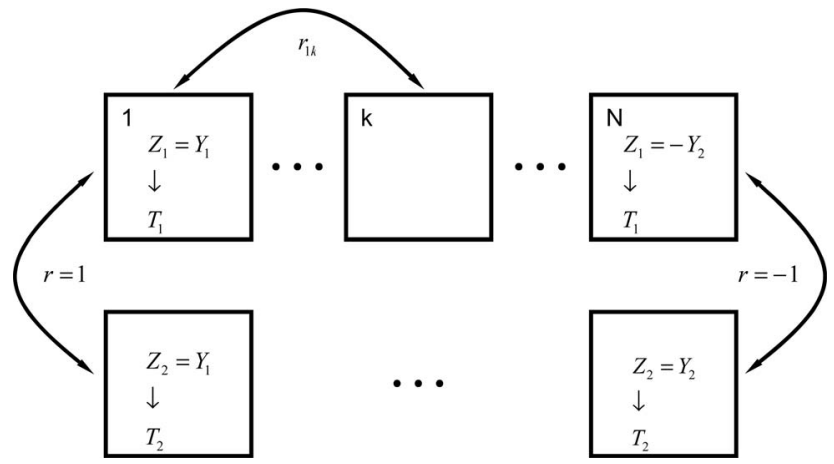

Fig. 2. Illustration of evolution of a correlated sequence of $N$ speckle patterns. Phase arrays $T_{1}$ and $T_{2}$ with degree of correlation $r$ are generated from bivariate Gaussian arrays $Z_{1}$ and $Z_{2}$, which in turn are generated from S.I. arrays $Y_{1}$ and $Y_{2}$. 


$$
r_{1 k} \equiv \frac{E\left\{\left(T_{11}-\mu_{11}\right)\left(T_{1 k}-\mu_{1 k}\right)\right\}}{\sigma_{11} \sigma_{1 k}}=\sqrt{\frac{1+r}{2}} .
$$

To provide the link between the specified correlation between the phase realizations and the speckle correlation coefficient, we inspect the variance of the phase difference [see Eq. (6)],

$$
\begin{aligned}
\operatorname{var}\left\{\phi_{1}-\phi_{2}\right\} & =(2 \pi)^{2} E\left\{\left[\left(T_{11}-\mu_{11}\right)-\left(T_{1 k}-\mu_{1 k}\right)\right]^{2}\right\} \\
& =(2 \pi)^{2}\left\{\sigma_{11}^{2}-2 \sigma_{11} \sigma_{1 k} r_{1 k}+\sigma_{1 k}^{2}\right\} \\
& =(2 \pi)^{2} 2 \sigma_{T}^{2}\left(1-r_{1 k}\right) \\
& =(2 \pi)^{2}\left(1-r_{1 k}\right) / 6
\end{aligned}
$$

where we have made use of the fact that $T$ is uniformly distributed and thus $\sigma_{T}^{2}=1 / 12$.

From the previous discussion we see that the speckle correlation coefficient is given by

$$
\rho_{1 k}=\exp \left\{-\frac{(2 \pi)^{2}}{6}\left(1-r_{1 k}\right)\right\},
$$

where we have denoted explicitly that the speckle correlation is referenced to the first realization in the sequence. If we wish the argument of the exponential to take the form of a power law,

$$
1-r_{1 k}=\left(\frac{k-1}{N-1}\right)^{\nu}
$$

we must specify the correlation coefficient for the phase realization pair as

$$
r=2\left[1-\left(\frac{k-1}{N-1}\right)^{\nu}\right]^{2}-1
$$

The speckle correlation coefficient then becomes

$$
\begin{gathered}
\rho_{1 k}=\exp \left\{-2\left(\frac{k-1}{c}\right)^{\nu}\right\}, \\
c=(N-1)\left(3 / \pi^{2}\right)^{1 \nu} .
\end{gathered}
$$

To better understand the generation of correlated phase, we rewrite the intermediate result before the percentile transformation [Eq. (5)] to the unit interval:

$$
\begin{gathered}
Z_{1}=\sqrt{-2 \ln X_{1}} \cos \left(2 \pi X_{2}+\phi\right), \\
\phi=\tan ^{-1}\left(\frac{\sqrt{1-r}}{\sqrt{1+r}}\right) .
\end{gathered}
$$

Here, it is perfectly clear that the RV, $Z_{1}$ as $r$ evolves between +1 and -1 is a pure sinusoid whose initial and final values are, respectively,

$$
\begin{gathered}
Z_{1}=\sqrt{-2 \ln X_{1}} \cos \left(2 \pi X_{2}\right), \\
Z_{1}=\sqrt{-2 \ln X_{1}} \cos \left(2 \pi X_{2}+\pi / 2\right) .
\end{gathered}
$$

If we inspect the specific case where $r$ is specified to give equal decorrelation increments,

$$
r=\cos \left(\pi \frac{k-1}{N-1}\right)
$$

then the incremental phase is given by

$$
\phi=\frac{\pi k-1}{2} \frac{k-1}{N-}
$$

Thus, the formula for generating a sequence of $Z$ values is

$$
Z_{1}(k)=\sqrt{-2 \ln X_{1}} \cos \left(2 \pi X_{2}+\frac{\pi k-1}{2} \frac{k-1}{N-} .\right.
$$

Upon performing the percentile transformation for the actual phase value and generation of the speckle pattern as prescribed earlier, one arrives at a sequence of patterns with correlation

$$
r_{1 k}=\cos \left(\frac{\pi k-1}{2} \frac{k-1}{N-}\right.
$$

From this, one observes that the algorithm produces physically realistic, continuous phase trajectories between the two limits of $r=1$ and $r=-1$ and that it requires only two arrays of random numbers to do so. This assertion follows from Eq. (17), and the subsequent step of the (continuous) percentile transformation [Eq. (5)]. As a result of this phase continuity, the evolution of the speckle pattern is also continuous, as one would expect from a real physical process [9]. Thus the procedure is distinct, for instance, from the algorithm of Federico et al. [2] and Rabal et al. [3], which adds a S.I. phase increment at each step in the sequence. Because the phase increment is discontinuous, so too is the evolution of the speckle pattern sequence.

\section{RESULTS}

Having described the procedure for generating a sequence of speckle patterns with prescribed correlation, we next proceed with a number of examples. In each case we generate a sequence of $N=50$ speckle patterns, each of 256 $\times 256$ pixels, and for a sampling $L / D=4$ that is twice the Nyquist criterion.

\section{A. Gaussian Speckle Correlation Coefficient}

For an exponent of $\nu=2$, Eq. (12) yields a sequential correlation function of

$$
\begin{aligned}
\rho_{1 k} & =\exp \left\{-2\left(\frac{k-1}{c}\right)^{2}\right\}, \\
c & =(N-1)\left(3 / \pi^{2}\right)^{1 / 2} .
\end{aligned}
$$

Results are shown in Figs. 3-6. Figure 3 is a display of the observed speckle pattern correlation coefficient, $\rho_{1 k}$, versus the prediction based on Eqs. (7) and (9). An alternative display of the observed speckle pattern correlation coefficient versus realization number is given in Fig. 4 along with the best-fit Gaussian and resulting parameter estimate, $c=26.59$. Note that this sequence is based on a single pair of random S.I. phase realizations and that the theoretical value of the parameter is $c=27.02$. Shown in 


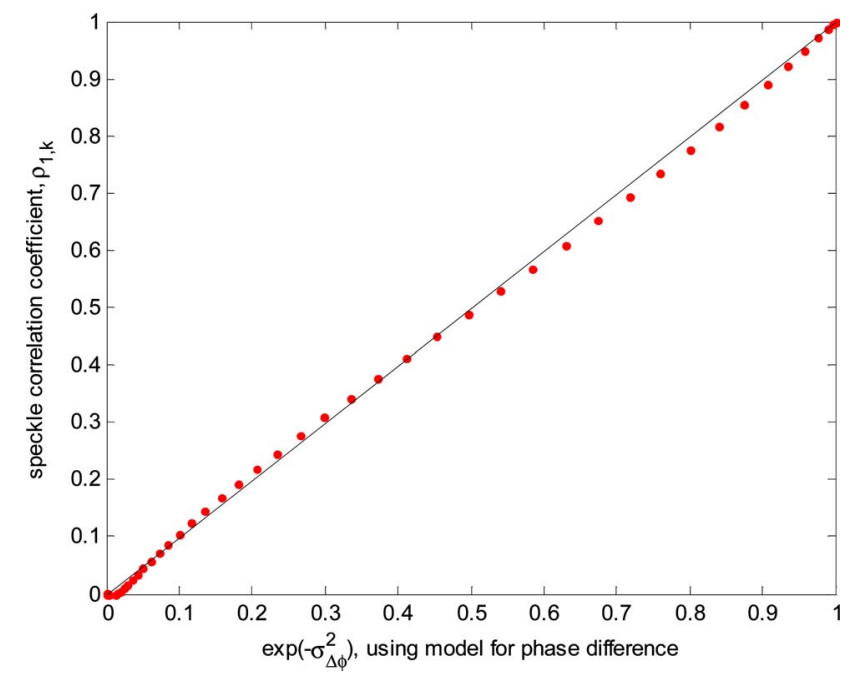

Fig. 3. (Color online) Calculated speckle correlation coefficient versus the model based on variance of the phase difference, for the specified Gaussian sequential correlation function.

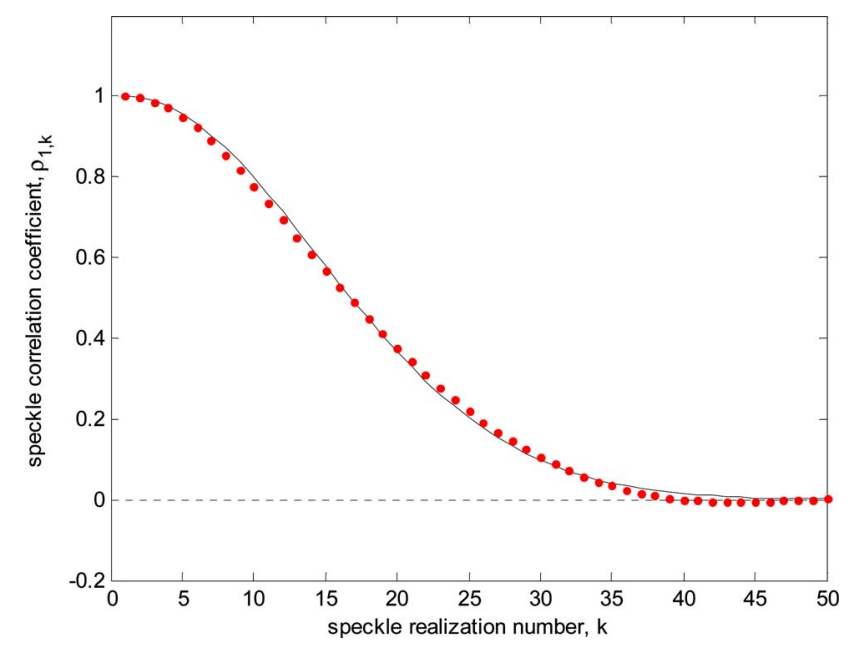

Fig. 4. (Color online) Speckle correlation coefficient as a function of sequence number, for the specified Gaussian sequential correlation function.

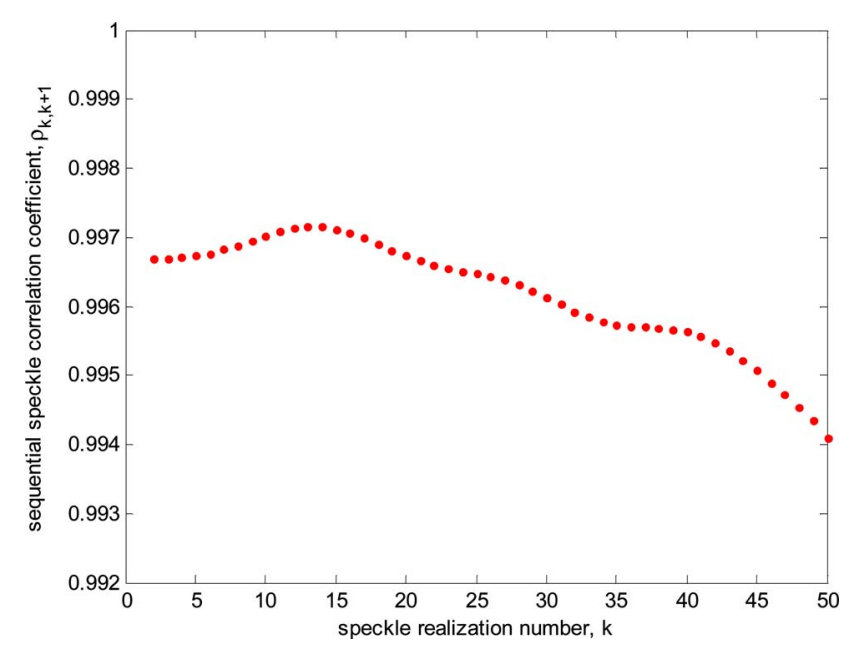

Fig. 5. (Color online) Sequential speckle correlation coefficient, for the specified Gaussian sequential correlation function.

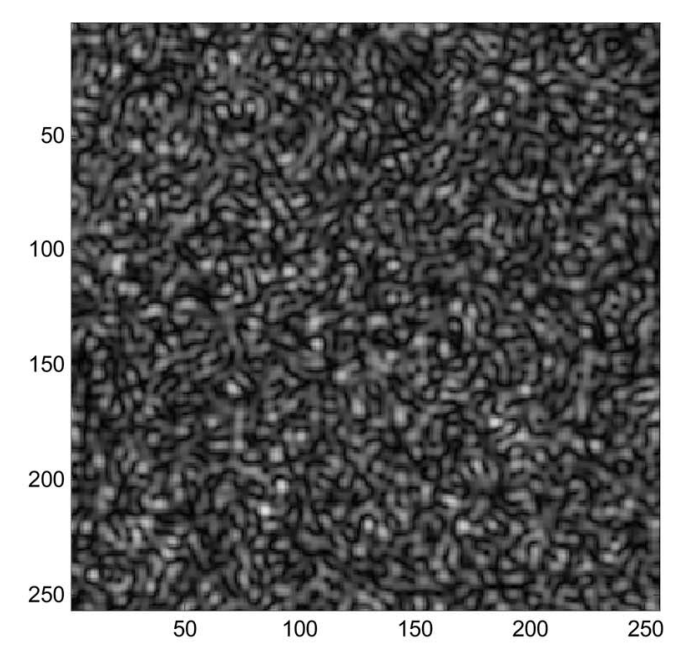

(a)
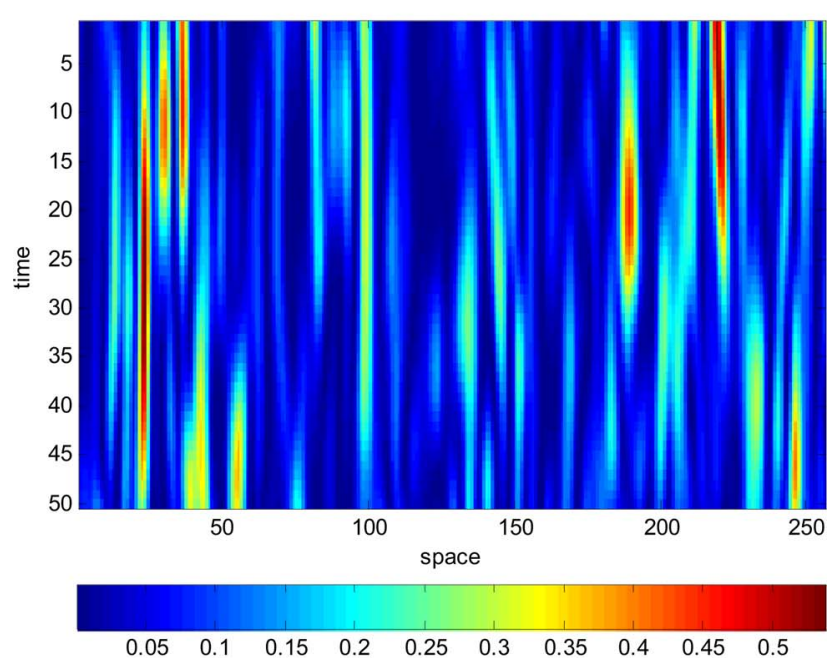

(b)

Fig. 6. (Color online) (a) First frame from the speckle realization cube. (b) Slice through the central row in the speckle cube illustrating the temporal continuity of the speckle pattern. The spatial dimension is along the horizontal axis, and time is along the vertical axis.

Fig. 5 is the observed speckle pattern correlation coefficient between successive realizations. A reasonable estimate of the mean value of the sequential speckle correlation coefficient was found to be given by

$$
E\left\{\rho_{k, k+1}\right\}=1-10 N^{-2} .
$$

The estimate so produced is 0.9960 versus the observed value of 0.9962 . Figure 6 (a) shows the initial speckle image from the cube of realizations, and Fig. 6(b) is a slice through this cube such that the spatial dimension is along the horizontal axis and time is along the vertical. This type of display seems to date to Oulamara et al. [9] and has been called the time history of the speckle pattern (THSP) [1] and, earlier, a stacked speckle history [10]. In other fields, notably radiography and fluorescence speckle microscopy [11], such a spatio-temporal display is called a kymograph. The specific results of Fig. 6(b) illustrate the claimed temporal continuity of the speckle pattern. 


\section{B. Exponential Speckle Correlation Coefficient}

Repeating the above procedure for $\nu=1$, we obtain the results shown in Figs. 7-9. Also shown in Fig. 8 is the bestfit exponential model with parameter $c=14.84$ versus the model-based value of $c=14.89$.

\section{Constant Speckle Correlation Coefficient}

An empirically derived correlation specification of

$$
r=\cos \left[\pi \frac{k-1}{N-1}\right]
$$

which yields a model-based estimate of the speckle correlation coefficient of

$$
\rho_{1 k}=\exp \left\{-\frac{(2 \pi)^{2}}{3} \sin ^{2}\left[\frac{\pi}{4}\left(\frac{k-1}{N-1}\right)\right]\right\},
$$

produces the results shown in Figs. 10-12. Note that the earlier comment about the phase realizations being $180^{\circ}$

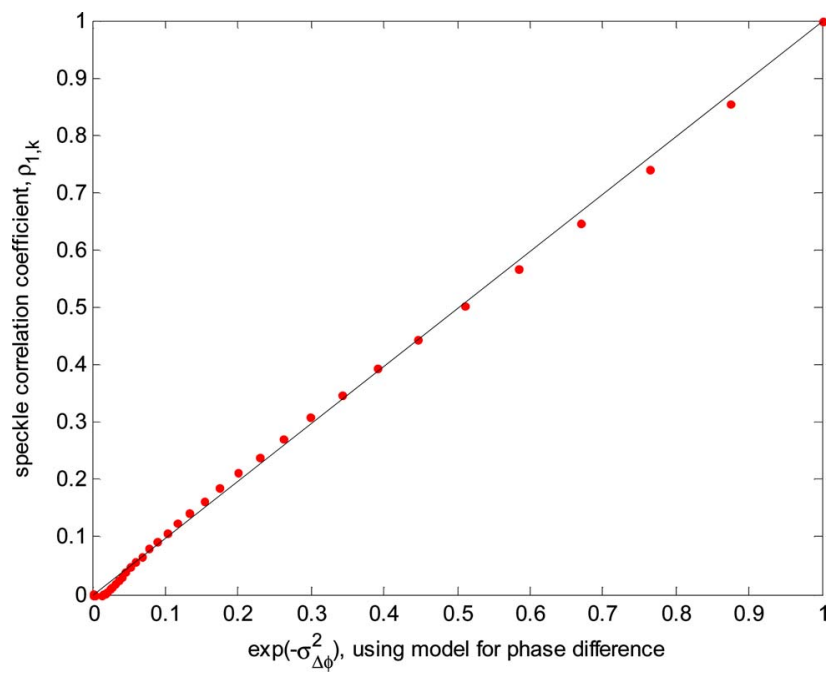

Fig. 7. (Color online) Calculated speckle correlation coefficient versus the model based on variance of the phase difference, for the specified exponential sequential correlation function.

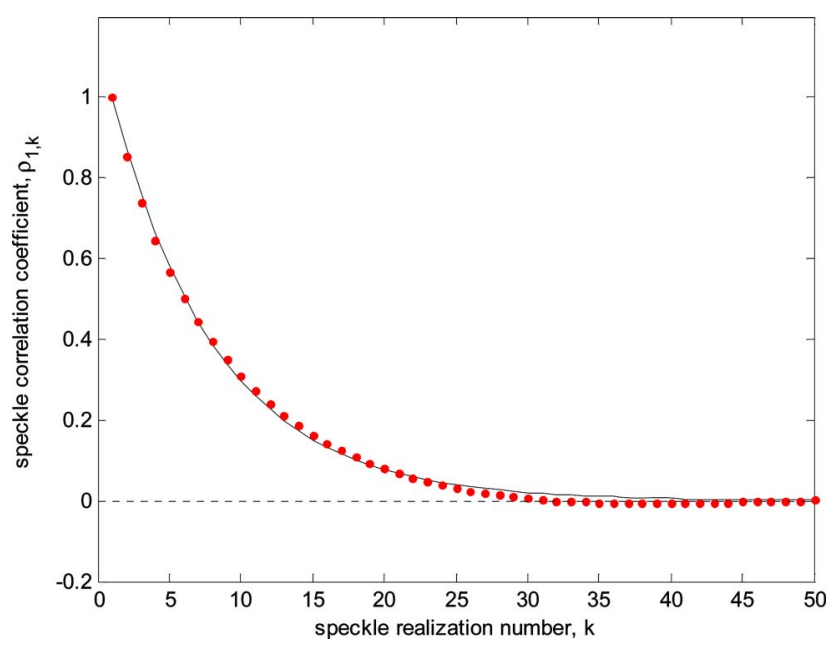

Fig. 8. (Color online) Speckle correlation coefficient as a function of sequence number, for the specified exponential sequential correlation function.

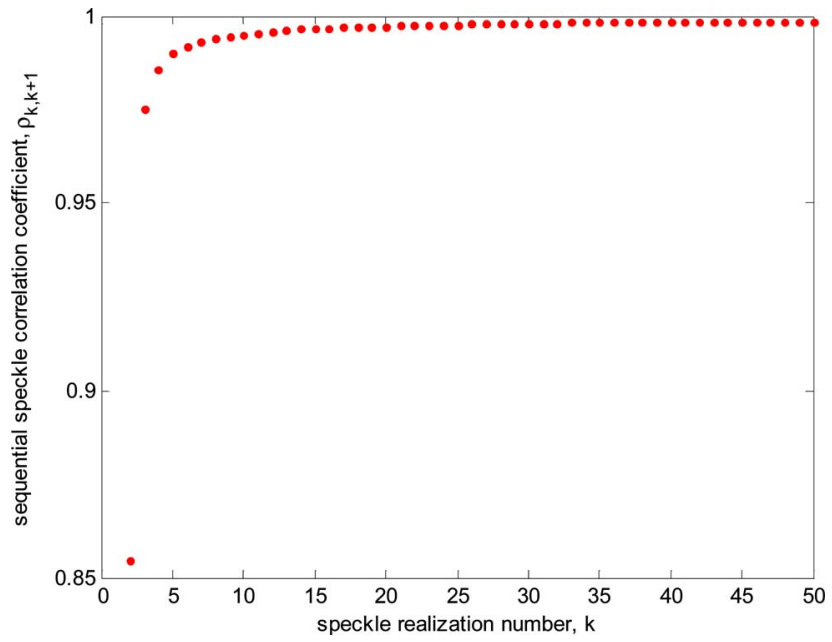

Fig. 9. (Color online) Sequential correlation coefficient for the exponential model, for the specified exponential sequential correlation function.

rotations of one another for $r=-1$ gives a hint for the form of relationship in Eq. (21). Also shown in Fig. 11 is the best-fit Gaussian model with parameter $c=24.60$.

In the previously discussed algorithm, the random phasor was of the form

$$
\exp \{i 2 \pi T\}
$$

where $T$ was uniformly distributed on $(0,1)$. Invoking the complex Gaussian moment theorem leads to the expression for the correlation between the first speckle realization in the sequence and the $k$ th realization, Eq. (9).

Now consider a random phasor of the form

$$
\exp \{i 2 \pi m T\}
$$

where $m$ is a multiplicative factor and $T$ is uniformly distributed on the unit interval as before. The resulting sequential correlation behavior is

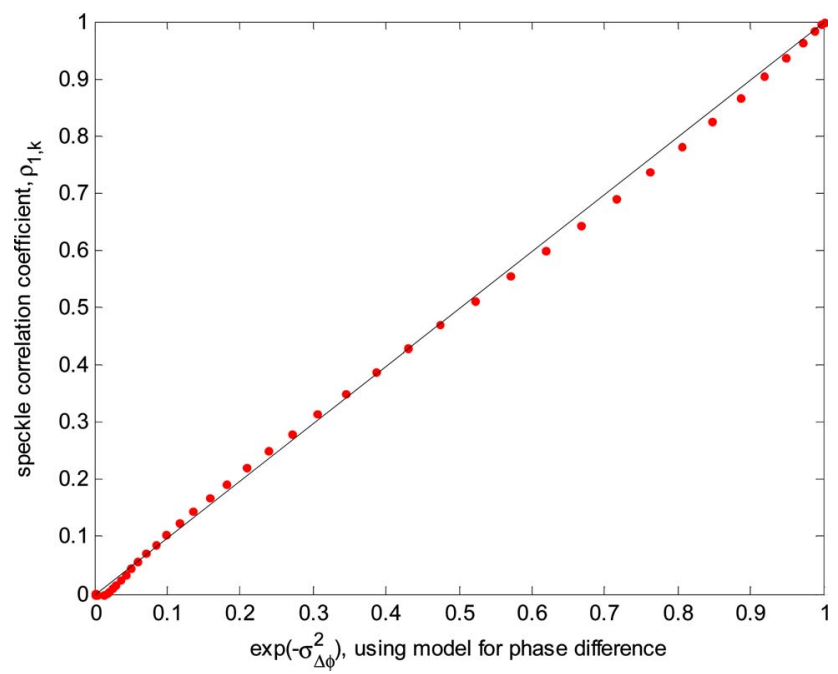

Fig. 10. (Color online) Speckle correlation coefficient versus the model based on variance of the phase difference, for the specified constant sequential correlation function. 


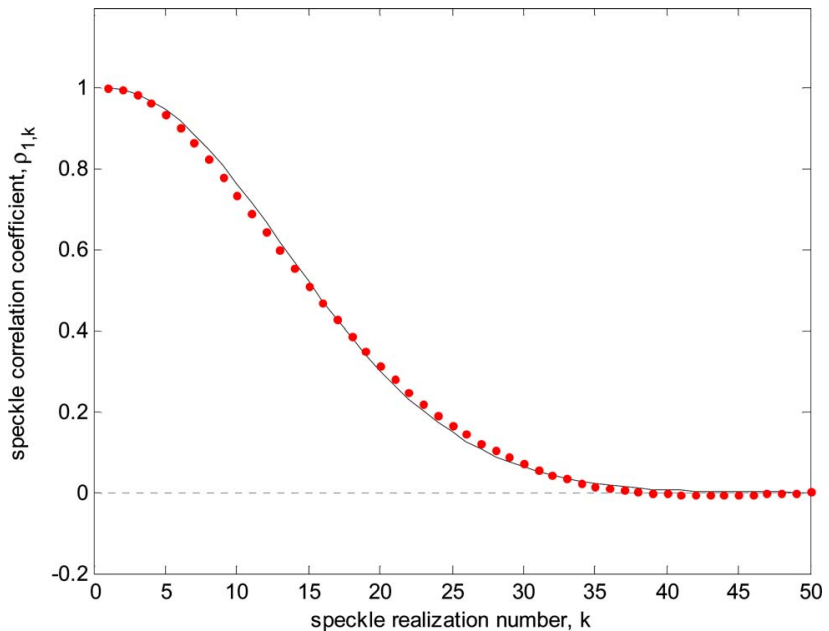

Fig. 11. (Color online) Speckle correlation coefficient as a function of sequence number, for the specified constant sequential correlation function.

$$
\rho_{1 k}=\exp \left\{-\frac{(2 \pi m)^{2}}{6}\left(1-r_{1 k}\right)\right\} .
$$

This approach allows for the generation of a speckle sequence that decorrelates from the initial realization within a prescribed time in a specified manner but retains a particular correlation between sequential realizations. As an example, consider the specification

$$
\begin{gathered}
m=3 \\
r_{1 k}=\cos \left(\frac{\pi}{2} \frac{k-1}{N-1}\right) .
\end{gathered}
$$

Results are shown in Figs. 13 and 14. As seen from these results, the multiplicative factor of 3 has shortened the correlation time by the corresponding amount (compare Figs. 11 and 13, for which the best-fit Gaussian parameters are, respectively, 24.60 and 8.43), but the desired sequential behavior is retained (compare Figs. 12 and 14). For this generalization, a reasonable estimate of the

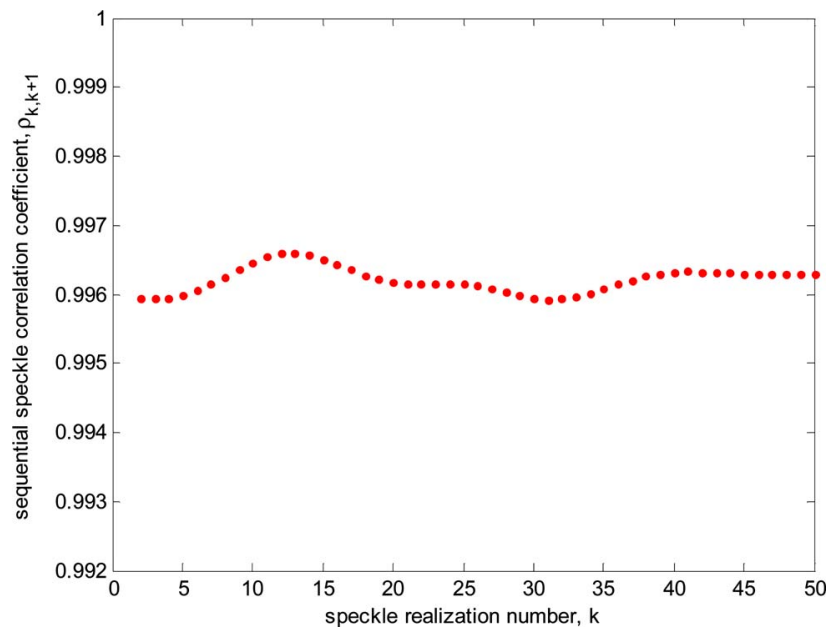

Fig. 12. (Color online) Sequential speckle polarization coefficient (mean is 0.9962 ), for the specified constant sequential correlation function. Note the constant observed value for this case.

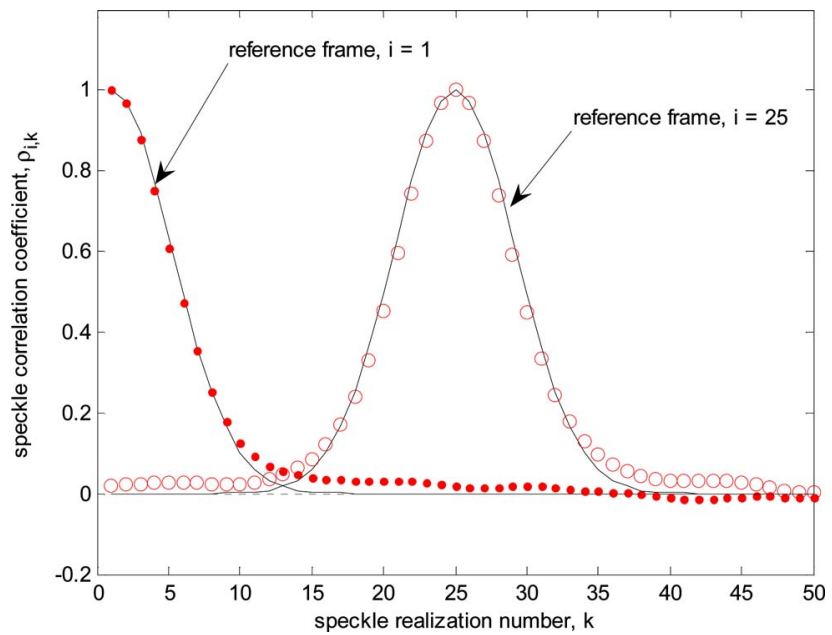

Fig. 13. (Color online) Speckle correlation coefficient as a function of sequence number for the multiplicative factor of $m=3$. Results are shown for frames 1 and 25 chosen as the reference, for the specified constant sequential correlation function.

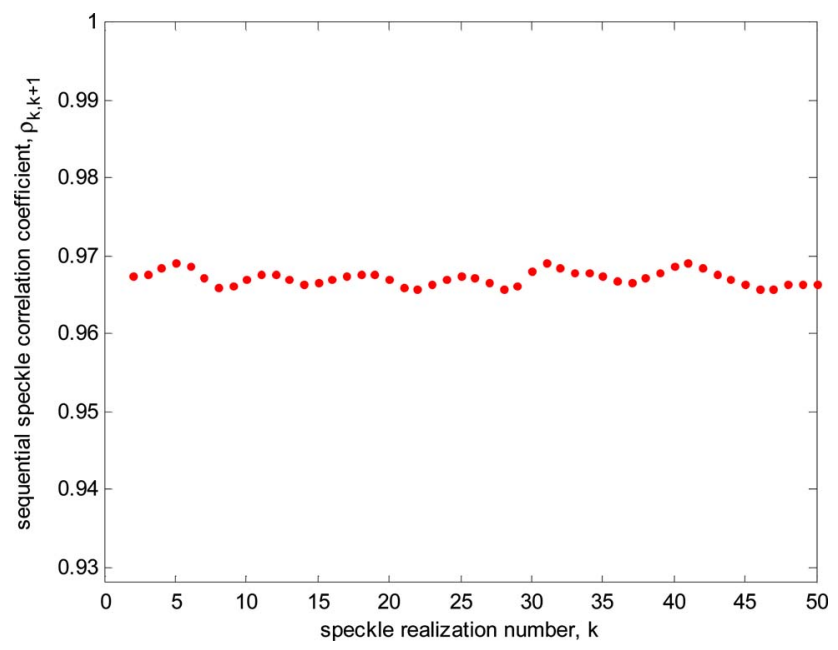

Fig. 14. (Color online) Sequential speckle polarization coefficient (mean is 0.9673 ), for the specified constant sequential correlation function and multiplicative factor of $m=3$.

mean sequential correlation is given by

$$
E\left\{\rho_{k, k+1}\right\}=1-10(m / N)^{2} .
$$

\section{DISCUSSION AND CONCLUSIONS}

We have illustrated the use of the concept of a copula to generate two representative functional forms for the decorrelation of an objective speckle sequence, Gaussian and exponential. The approach, however, is not limited to monotonically decreasing functions such as these. Moreover, much more complex dependencies could be imagined. As an example, for the case in which both ordered and unordered motion of the scatterers is present, one could expect to see the product of these two functional forms. Note that the exponential corresponds to a Lorentzian temporal power spectrum (homogeneously broadened line), while a Gaussian correlation function of course corresponds to a Gaussian (inhomogeneously broadened line). The product of the exponential and Gaussian forms 
corresponds to a convolution of the line profiles, producing a Voigt profile [12]. Such a functional form is easily prescribed using the procedures established herein.

Extensions. An effect seen in OCT images of highly scattering media is the slow depolarization with depth. Near the surface, the speckle statistics are exponentially distributed, as one would expect for polarized speckle. As the depth increases, however, scatter produces an orthogonally polarized component of the measured signal. As a result, the speckle statistics become Rayleighdistributed (due to the incoherent addition of two uncorrelated speckle patterns, one for each of the polarization components). A simple way to simulate this effect is to use the preceding algorithm to generate a pair of speckle image cubes such that [see Eq. (13)]

$$
\text { cube1: } \begin{aligned}
Z_{1} & =\sqrt{-2 \ln X_{1}} \cos \left(2 \pi X_{2}+\phi\right), \\
\text { cube2: } Z_{1} & =\sqrt{-2 \ln X_{1}} \cos \left(2 \pi X_{3}+\phi\right), \\
\phi & =\tan ^{-1}\left(\frac{\sqrt{1-r}}{\sqrt{1+r}}\right),
\end{aligned}
$$

where $X_{1}, X_{2}$, and $X_{3}$ are mutually S.I. and uniformly distributed. In this manner, each cube begins with the same speckle pattern but each evolves toward a different (S.I.) pattern. If one adds the two image cubes frame by frame, then the first frame of the resulting cube is simply the single (exponentially distributed) speckle pattern. The last frame in the sequence is the sum of two S.I. speckle realizations and is thus Rayleigh-distributed. The frames in the sequence slowly evolve from the initial exponential to the final Rayleigh distribution. A slice through this cube for a given column or row would then constitute what is commonly referred to as an OCT image or B-scan [13].

This study has been restricted to the generation of objective speckle patterns. A similar approach [14] can be used to generate subjective speckle patterns via the algorithm

$$
I=\left|F^{-1}\{H \cdot F\{\exp (i \phi)\}\}\right|^{2},
$$

where $F$ denotes the Fourier transform, $H$ represents the pupil of the imaging system, and $\phi$ is the random phase realization. The issue with subjective speckle is that it is not obvious that the complex Gaussian moment theorem holds for such a few-to-one mapping. It is valid for a statistical average, but even if one invokes ergodicity, the few-to-one mapping makes this a questionable argument. It should be clear from the theory described herein, however, that an appeal to ergodicity has not been made. Rather, the algorithms put forth generate a single sample function of the random speckle process. As such, one could imagine a numerical study in which these issues might be explored.
As a general comment about the use of the copula concept, we note that the final step in the algorithm for generating uniformly distributed phases [Eq. (5)] is arbitrary. One could just as easily use two different (non-Gaussian) cumulative distribution functions to generate arbitrarily distributed (but correlated) instantiations. Such a procedure has been used for example, in Monte Carlo studies involving finance [15]. Perhaps it is time for the optics community to embrace this concept.

\section{ACKNOWLEDGMENTS}

This work was sponsored in part by National Institutes of Health grant CA103824.

\section{REFERENCES}

1. H. J. Rabal, R. Arizaga, N. L. Cap, E. Grumel, and M. Trivi, "Numerical model for dynamic speckle: an approach using the movement of the scatterers," J. Opt. A, Pure Appl. Opt. 5, S381-S385 (2003).

2. A. Federico, G. H. Kaufmann, G. E. Galizzi, H. Rabal, M. Trivi, and R. Arizaga, "Simulation of dynamic speckle sequences and its application to the analysis of transient processes," Opt. Commun. 260, 493-499 (2006).

3. H. J. Rabal, N. L. Cap, M. Trivi, R. Arizaga, A. Federico, G. E. Galizzi, and G. H. Kaufmann, "Speckle activity images based on the spatial variance of the phase," Appl. Opt. 45, 8733-8738 (2006)

4. J. W. Goodman, Statistical Optics (Wiley, 1985).

5. R. B. Nelson, An Introduction to Copulas (Springer-Verlag, 1999).

6. R. Bandyopadhyay, A. S. Gittings, S. S. Suh, P. K. Dixon, and D. J. Durian, "Speckle-visibility spectroscopy: a tool to study time-varying dynamics," Rev. Sci. Instrum. 76, 093110 (2005).

7. A. Papoulis and S. U. Pillai, Probability, Random Variables and Stochastic Processes, 4th ed. (McGraw-Hill, 2002).

8. J. W. Goodman, "Statistical properties of laser speckle patterns," in Laser Speckle and Related Phenomena, J. C. Dainty, ed. (Springer-Verlag, 1975), pp. 9-75.

9. A. Oulamara, G. Tribillon, and J. Duvernoy, "Biological activity measurement on botanical specimen surfaces using a temporal decorrelation effect of laser speckle," J. Mod. Opt. 36, 165-179 (1989).

10. D. D. Duncan, F. F. Mark, and L. W. Hunter, "A new speckle technique for noncontact measurement of small creep rates," Opt. Eng. 31, 1583-1589 (1992).

11. L. Ji and G. Danuser, "Tracking quasi-stationary flow of weak fluorescent signals by adaptive multi-frame correlation," J. Microsc. 220, 150-167 (2005).

12. M. E. Thomas and D. D. Duncan, "Atmospheric transmission," in Atmospheric Propagation of Radiation, Vol. 2 of The Infrared and Electro-Optical Systems Handbook, F. G. Smith, ed. (ERIM Infrared Information Analysis Center and SPIE, 1993).

13. S. R. Sadda, S. Joeres, Z. Wu, P. Updike, P. Romano, A. T. Collins, and A. C. Walsh, "Error correction and quantitative subanalysis of optical coherence tomography data using computer-assisted grading," Invest. Ophthalmol. Visual Sci. 48, 839-848 (2007).

14. J. W. Goodman, Introduction to Fourier Optics, 3rd ed. (Roberts, 2005).

15. U. Cherubini, E. Luciano, and W. Vecchiato, Copula Methods in Finance (Wiley, 2004). 